\title{
Exercise-Enhanced ICG Lymphography: A Fast Approach to Diagnosis and Staging of Lymphedema
}

\author{
Wei F. Chen, MD, FACS ${ }^{*}$; Evelyn S. Qin, MD²; Mindy J. Bowen, RN, BSN, CAPA3; Amy S. Little, DPT; \\ Jonathan N. Lensing, $\mathrm{MD}^{3}$ \\ ${ }^{1}$ Center for Lymphedema Research and Reconstruction, Department of Plastic Surgery, Cleveland Clinic, Cleveland, Ohio, USA \\ ${ }^{2}$ Department of Rehabilitation Medicine, University of Washington, Seattle, Washington, USA \\ ${ }^{3}$ Department of Surgery, University of lowa Hospitals and Clinics, lowa City, lowa, USA
}

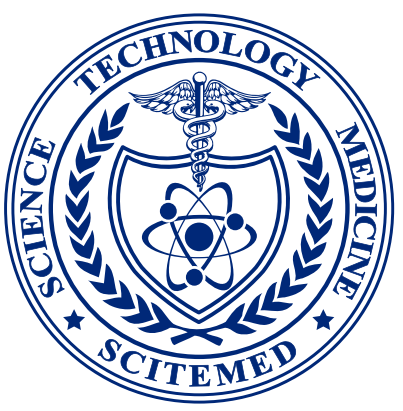

\begin{abstract}
Background: Indocyanine green (ICG) lymphography has been relied upon extensively in lymphedema management. Its utility extends from diagnosis and severity staging to treatment planning and outcome tracking. When performing ICG lymphography, a delayed scan following ICG injection is necessary due to the delayed emergence of lymphographic dermal backflow patterns. The need for delayed scan, however, makes ICG lymphography a time-consuming study. We conducted a prospective study with the primary goal to decrease the overall study time by applying the known phenomenon of exercise-induced lymphatic acceleration, and the secondary goal to further improve the study's accuracy and reproducibility.

Methods: Following standard ICG injections, patients were exercised on a recumbent cross-trainer at five-minute intervals at specific, controlled intensity. Delayed scanning was performed following each exercise interval, and sequential changes of lymphographic patterns were recorded. The cycle of exercise/ scan continued until achieving plateau, or when no further lymphographic changes were observed for two consecutive cycles. Following the point of the plateau, further delayed scanning was performed every hour to identify the time point when the lymphographic patterns started to recede. Twenty-three limbs in 9 patients (10 arms, 13 legs) with unilateral and bilateral lymphedema were studied.

Results: Following ICG injection, the lymphographic patterns evolved continually until plateauing after three cycles of exercise (15 minutes of exercise in total) in all limbs studied, and the dye was shown to start receding after 4 hours. Patients preferred exercising to speed up ICG studies compared to the traditional method which involves waiting between six to 14 hours between initial and delayed ICG lymphography scans.

Conclusion: ICG lymphography is the current gold standard in lymphedema diagnosis. The study can be accelerated to only taking 15 minutes while further increasing the accuracy and reproducibility of the study with the addition of exercise.
\end{abstract}

\section{INTRODUCTION}

Indocyanine green (ICG) lymphography is a non-radioactive, fluorophore-based imaging modality that provides functional imaging of the lymphatic system. With specificity comparable to and sensitivity superior to technetium ${ }^{99}$-based lymphoscintigraphy, the conventional diagnostic gold standard, ICG lymphography has been increasingly relied upon to diagnose extremity lymphedema. The diagnosis of lymphedema is confirmed when the pathologic dermal backflow patterns are seen. In addition to providing a definitive diagnosis, the types/distribution of the dermal backflow patterns presently allows the determination of disease severity. Since the dermal backflow patterns emerge in a delayed fashion and, after their initial emergence, they continually evolve until reaching a steady-state, or plateau, a delayed scan at the time of plateau is necessary to achieve accurate representation of the disease. Exercise has been shown to accelerate lymphatic drainage $[1,2]$. Based on this phenomenon, we aimed to 1) determine if controlled exercise could shorten the time it takes for ICG lymphography to reach a plateau, and to 2) create a standardized protocol to increase efficiency and reliability of ICG lymphography to diagnose extremity lymphedema.

\section{METHODS}

\section{Participants}

With approvals from the Institutional Review Board and National Insti- tutes of Health's Clinical Trial Registry, all lymphedema patients presented to our lymphedema reconstruction center over a 6-month period from April to September 2019 who met inclusion criteria were recruited into the study. Inclusion criteria included being 18 years or older, having voluntarily agreeing to participate in the study, being suspected to have lymphedema or previously diagnosed with lymphedema. Exclusion criteria included having an allergy to iodine, women who were pregnant or nursing, having heart/lung/joint conditions that would prevent exercising. Campisi criteria were used to clinically stage disease severity.

\section{Exercise-Enhanced ICG-Lymphography Protocol}

Depending on the limb affected, $0.1 \mathrm{~mL}$ of $0.25 \%$ ICG (Akorn Pharmaceutical, Lake Forest, IL) was injected into two interdigital spaces and wrist (arm) or medial malleolus (leg). Following injection, baseline lymphatic uptake, and pump function before exercise were evaluated with immediate scanning using SPY Elite (Stryker, Kalamazoo, MI). The pump function was evaluated by the time required for ICG to reach antecubital fossa/ axilla and popliteal fossa/groin. The test subject then began exercising on a recumbent cross trainer (Video 1: https://youtu.be/ftMge4ePwDI) at 5-minute intervals, with ICG scanning interspersed between two exercise intervals, until the lymphographic plateau was reached. Lymphographic plateau was defined as having no observable changes in lymphographic patterns in three consecutive scans. The exercise intensity was controlled at rate perceived exertion (RPE) of 12-13, or moderate intensity. Study participants were asked to maintain RPE of 12-13, which is considered a rate perceived exertion described as between "light" to "somewhat hard". The approximate heart rate can be approximated for each level of activity by 


\begin{tabular}{|c|c|c|}
\hline Rating & Description of Perceived Exertion & Examples \\
\hline 6 & No exertion at all & Watching television, reading \\
\hline 7 to 8 & Extremely Light & Tying shoes \\
\hline 9 to 10 & Very Light & Light chores such as folding laundry \\
\hline 11 to 12 & Light & Walking or activities that require some effort but do not increase your breathing \\
\hline 13 to 14 & Somewhat Hard & Brisk walking/activities that increase heart rate and breathing, but does not make you short of breath \\
\hline 15 to 16 & Hard (Heavy) & Biking, swimming, or activities that take significant effort and gets the heart racing and makes you short of breath \\
\hline 17 to 18 & Very Hard & Highest level of activity you can sustain \\
\hline 18 to 19 & Extremely Hard/maximal exertion & Finishing a race or burst of activity that can't be sustained \\
\hline
\end{tabular}

\begin{tabular}{|l|l|}
\hline \multicolumn{2}{|l|}{ Table 2. Patient and Limb Characteristics } \\
\hline \multicolumn{1}{|l}{ Total Patients } \\
\hline Sex & 9 \\
\hline \multicolumn{1}{|l}{ Male } & $0 / 9$ \\
\hline Female & $9 / 9$ \\
\hline Age (in years) & $56.6 \pm 13.7$ \\
\hline Total Limbs Tested & 23 \\
\hline Right Upper Extremity & $5 / 23$ \\
\hline Left Upper Extremity & $5 / 23$ \\
\hline Right Lower Extremity & $7 / 23$ \\
\hline Left Lower Extremity & $6 / 23$ \\
\hline Diseased Limbs & $13 / 23$ \\
\hline Right Upper Extremity & $3 / 13$ \\
\hline Left Upper Extremity & $3 / 13$ \\
\hline Right Lower Extremity & $3 / 13$ \\
\hline Left Lower Extremity & $4 / 13$ \\
\hline Secondary Lymphedema & $13 / 13$ diseased limbs \\
\hline Plateau Time Average (in minutes) & $11.25 \pm 2.26$ \\
\hline Upper Extremity & $11.00 \pm 2.24$ \\
\hline Lower Extremity & $11.42 \pm 2.44$ \\
\hline
\end{tabular}

multiplying the Borg score by 10 (Table 1). After reaching the lymphographic plateau, scanning occurred at a one-hour interval until regression of the lymphographic patterns was observed, with the first of the hourly scanning was performed at 1-hour following ICG injection.

\section{Data Analysis}

Video footages of indocyanine green lymphography were independently reviewed by three experienced ICG lymphographers to identify time points of plateau and regression. SPSS (IBM Statistics Package for the Social Sciences, v25)e was used to perform the data analysis and performed using a significance level of 0.05 and a confidence interval of $95 \%$. Descriptive analysis generated frequencies of the study variables, and comparisons of categorical variables were performed using the chi-square test.

\section{RESULTS}

Nine patients and 23 limbs were recruited into the study-13 lymphede- ma-affected limbs and 10 normal limbs. The patients (mean age: $56.6 \pm$ 13.7 years old) consisted of nine females and no males (Table 2). Five patients had unilateral lymphedema and four had bilateral disease. All patients had secondary lymphedema-five were secondary to breast cancer, three were related to gynecologic cancer, and one was due to traumatic injury. Of the 13 diseased limbs, 6 limbs were staged as Campisi stage I, 4 limbs as Campisi stage II, and 3 with Campisi stage III. Nine of the 23 limbs had undergone lymphedema surgery with either lymphaticovenous anastomosis (LVA) or vascularized lymph vessel transfer (VLVT). When comparing patients with unilateral versus bilateral disease, no significant difference was seen by sex, age, BMI, and disease etiology. All patients successfully sustained RPE of 12-13 throughout the exercise phase, regardless of BMI.

In normal limbs, $80 \%$ of the limbs ( 8 of 10 ) demonstrated ICG passing through the antecubital fossa rapidly and reaching the axilla/groin during the initial baseline scan. The same $80 \%$ of the limbs plateaued during the initial baseline scanning prior to the initiation of exercise. No further changes in ICG lymphographic patterns were observed with exercises, $20 \%$ ( 2 of 10 ) of the normal limbs showed ICG reaching the antecubital fossa after 1 cycle of exercise (5 minutes) and reached axilla and plateaued following 2 cycles of exercises ( 10 minutes).

In lymphedematous limbs, interestingly, all (13 of 13) showed ICG reaching antecubital/popliteal fossa during the immediate baseline scan. The velocities at which they reach antecubital/popliteal fossa were found to be correlating to Campisi staging, with higher severity showing slower transit velocity (Figure 1). None plateaued during the baseline scan. All (13 of 13) plateaued following 3 cycles of exercise (15 minutes).

In all limbs, including both healthy and diseased limbs, the lymphographic patterns began to dissipate at four hours (Figure 2). The dye regression was independent of disease severity.

\section{DISCUSSION}

Indocyanine green lymphography has been increasingly performed for diagnosis, severity staging, treatment planning, intra-operative guidance, and post-operative tracking of lymphedema. However, due to the absence of a standardized protocol, the study result is not transferrable-the result of a study performed at one institution is not interpretable at another institution. Current methods either do not perform delayed scans at all, which incomplete and inaccurate assessment of pathology, or utilizes up to a 24-hour waiting period between immediate and delayed scanning $[3,4]$. Previously, we identified the need to perform a delayed scan at 6 hours for proper evaluation[5,6] and had successfully adopted this practice as part of our standardized diagnostics. While the 6-hour approach was time-tested in our practice, it was time-consuming, and it limited the number of patients we could evaluate in a clinic day.

Realizing this shortcoming of ICG lymphography, researchers attempted to accelerate lymph flow and thereby shorten the study time. 


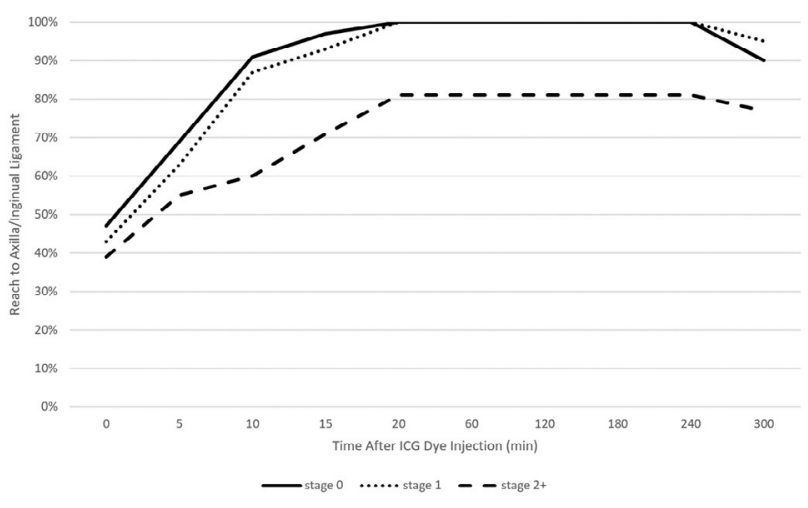

Figure 1. Average transit time of ICG dye $(0.1 \mathrm{~mL}$ of $0.25 \%$, Akorn Pharmaceutical, Lake Forest, IL) from hand or foot to axilla or inguinal ligament respectively, based on Campisi severity of lymphedema. Time zero is the time at which the limb was first injected with dye. Note, the ICG dye plateaus slightly after 15 minutes due to the time added to perform ICG scans in between each five-minute cycle of exercise. The dye then recedes approximately four hours after injection. ICG, indocyanine green.
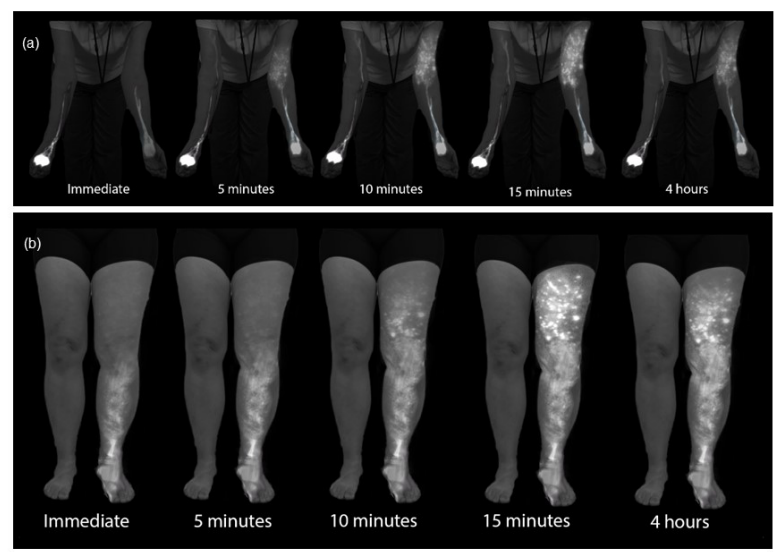

Figure 2. ICG patterning and transit along exercise timepoints in a healthy right arm, diseased left arm (a), and diseased left leg (b). The lymphatic pattern is seen almost immediately, but the entire pattern is not visualized until three rounds of exercise, illuminating the importance of a delayed scan. The pattern regresses by four hours. ICG, indocyanine green.

Suami et al. [7] applied manual lymphatic drainage (MLD) to accelerate the lymph flow following ICG injection. They were able to achieve lymphographic plateau at a promising 30-45 minutes. Despite the favorable result, it is impractical to require a skilled therapist to consistently perform MLD for an extended duration of time for all ICG studies. Matsumoto et al. [8] attempted to accelerate the study time by having patients walking on a treadmill at $2 \mathrm{~km} /$ hour. They concluded that twenty minutes of exercise is appropriate for lymphedema screening. This is relatively consistent with our findings. However, the study did not include patients with arm lymphedema and therefore would not be applicable to many of our patients who suffer from breast cancer-related lymphedema.

We selected recumbent cross trainer as our method of choice to accelerate lymph flow due to 1) it simultaneous exerts both upper and lower extremities, and 2) it accommodates patients of all sizes, fitness level, and functional capacities [9]. To control the extent at which the lymph flow was accelerated, we controlled the exercise intensity at RPE of 12-13. Borg's RPE is a validated tool for monitoring exercise intensity independent of age, gender, exercise type, coronary artery disease status, weight, fitness level, or functional ability[10,11]. RPE of 12-13 was chosen based on evi- dence showing this exercise intensity being safe and sustainable for prolonged exercise regardless of health levels $[12,13]$.

In this study, we were able to achieve a lymphographic plateau in all lymphedema-compromised limbs after 15 minutes of exercise at RPE of 12-13 on the recumbent cross-trainer. Interestingly, even in healthy limbs, although $80 \%$ achieved plateau rapidly during the initial baseline scanning without exercise, $20 \%$ still required 10 minutes of standardized exercise to reach a plateau. The above findings underscore the importance of delayed scanning for proper visualization and assessment of lymphatic physiology/pathology. They also confirmed our hypothesis that taking advantage of exercise-induced acceleration of lymph flow, ICG lymphography can be performed in an expedited fashion. The time to a plateau of 15 minutes compares highly favorably to our current practice of 6 hours. In addition to increased efficiency, this protocol would also increase the consistency and reliability of the study by controlling the patient's activity level between the immediate and delayed scans. In our current practice, we do not, and would not be able to even if we desired to do so, control patient's activity during the 6-hour interval between the immediate and delayed scans. We have long recognized this as a factor that introduced inaccuracy to our ICG lymphography. This exercise-enhanced protocol would resolve the above-mentioned problem.

An insufficient amount of wait time between the immediate and delayed scans would result in an inaccurate result. By the same token, excessive wait time causes problems as well. This was demonstrated by our identification of ICG regression in all cases at 4 hours following injection. This suggested that all scanning performed beyond 4 hours would result in the under-visualization of pathology.

From a practical standpoint, the implementation of a recumbent cross trainer exercise machine into the ICG lymphography protocol will involve an initial financial investment of $\$ 4,000-\$ 6,000$ USD per recumbent cross-trainer. This cost would quickly be offset with this accelerated protocol, as the estimated number of patients that could be evaluated would increase from 4 to approximately 10 patients a day, with each ICG visit producing 2.36 RVUs per scan (CPT code 38790 ). Due to our highly favorable experience with this exercise-enhanced protocol, we have adopted this new, more efficient, and reliable ICG lymphography protocol in our center as our standard practice.

\section{CONCLUSION}

ICG lymphography is an invaluable tool in lymphedema management. Both immediate and delayed scans are needed when performing the study. The delayed scan needs to be performed at the time of the lymphographic plateau to appreciate the full extent of the pathology. Using a recumbent cross trainer, the lymphographic plateau can be achieved in 15 minutes following ICG injection. We have found this exercise enhanced ICG lymphography protocol worthwhile of adoption by high volume lymphedema centers to raise diagnostic accuracy and efficiency.

\section{ARTICLE INFORMATION}

*Correspondence: Wei F. Chen, MD, FACS. Center for Lymphedema Research and Reconstruction Department of Plastic Surgery, Cleveland Clinic, 9500 Euclid Ave, Desk A60 Cleveland, Ohio 44195, USA. Email weifchen@hotmail.com

Received: Oct. 09, 2020; Accepted: Apr. 05, 2020; Published: May 31, 2021

DOI: 10.24983/scitemed.imj.2021.00140

Ethics Approval and Consent to Participate: The study is in accordance with the ethical standards of the 1964 Helsinki declaration and its later amendments or comparable ethical standards. This study was approved by and conducted in 


\section{ORIGINAL}

compliance with the University of lowa Institutional Review Board.

Informed Consent: Informed consent was obtained from the patient for publication of this Case report and any accompanying images.

Disclosures: This study was presented at International Society of Physical and Rehabilitation Medicine, on March 2020 in Orlando, Florida, USA.

Funding: The study did not receive any specific grant from funding agencies in the public, commercial, or not-for-profit sectors.

Conflict of Interest: The authors report no financial or other conflict of interest relevant to this article, which is the intellectual property of the authors.

Copyright @ 2021 The Authors. This is an open-access article distributed under the terms of the Creative Commons Attribution 4.0 International License (CC-BY).

\section{REFERENCES}

1. Desai P, Williams AG, Jr., Prajapati P, Downey HF. Lymph flow in instrumented dogs varies with exercise intensity. Lymphat Res Biol 2010;8(3):143-148.

2. Downey HF, Durgam P, Williams AG, Jr., Rajmane A, King HH, Stoll ST. Lymph flow in the thoracic duct of conscious dogs during lymphatic pump treatment, exercise, and expansion of extracellular fluid volume. Lymphat Res Biol 2008;6(1):3-13.

3. Yamamoto T, Yoshimatsu H, Narushima M, Yamamoto N, Hayashi A, Koshima I. Indocyanine green lymphography findings in primary leg lymphedema. Eur J Vasc Endovasc Surg 2015;49(1):95-102.

4. Narushima M, Yamamoto T, Ogata F, Yoshimatsu H, Mihara M Koshima I. Indocyanine green lymphography findings in limb lymphedema. J Reconstr Microsurg 2016;32(1):72-79.

5. Chen WF, Zhao H, Yamamoto T, Hara H, Ding J. Indocyanine green lymphographic evidence of surgical efficacy following microsurgical and supermicrosurgical lymphedema reconstructions. J Reconstr Microsurg 2016;32(9):688-698.

6. Jones G.E. and Hall-Findlay E.J.: Reduction Mammaplasty. In: Jones GE, editor. Bostwick's plastic \& reconstructivebreast surgery. New York: Thieme Publishers, 2010.

7. Suami H, Heydon-White A, Mackie H, Czerniec S, Koelmeyer L, Boyages J. A new indocyanine green fluorescence lymphography protocol for identification of the lymphatic drainage pathway for patients with breast cancer-related lymphoedema. BMC Cancer 2019;19(1):985.

8. Matsumoto K, Shinaoka A, Yamada K, Kimata Y. Exercise-loaded indocyanine green fluorescence lymphangiography for diagnosing lymphedema. J Reconstr Microsurg 2019;35(2):138-144.

9. Peterson MD, Lukasik L, Muth $\mathrm{T}$, et al. Recumbent cross-training is a feasible and safe mode of physical activity for significantly motor-impaired adults with cerebral palsy. Arch Phys Med Rehabil 2013;94(2):401-407.

10. Scherr J, Wolfarth B, Christle JW, Pressler A, Wagenpfeil S, Halle M. Associations between Borg's rating of perceived exertion and physiological measures of exercise intensity. Eur J Appl Physiol 2013;113(1):147-155.

11. Chen MJ, Fan X, Moe ST. Criterion-related validity of the Borg ratings of perceived exertion scale in healthy individuals: a meta-analysis. J Sports Sci 2002;20(11):873-899.

12. King AC, Haskell WL, Taylor CB, Kraemer HC, DeBusk RF. Group- vs home-based exercise training in healthy older men and women. A community-based clinical trial. JAMA 1991;266(11):1535-1542.

13. Borg GA. Psychophysical bases of perceived exertion. Med Sci Sports Exerc 1982;14(5):377-381. 